\title{
Effet De Prétraitements, De Substrats Et De Stress Hydriques Sur La Germination Et La Croissance Initiale De Diospyros Mespiliformis Hochst. Ex A.DC
}

\author{
Ali Ado \\ Université d'Agadez, Faculté des Sciences et Techniques, \\ BP 199 Agadez, Niger, \\ Université Abdou Moumouni de Niamey, Faculté des Sciences et \\ Techniques, Département de Biologie Laboratoire Garba Mounkaila, \\ BP 10662, Niamey, Niger \\ Issoufou Hassane Bil-Assanou \\ Université Dan Dicko Dankoulodo de Maradi, \\ Faculté d'Agronomie et des Sciences de l'Environnement, \\ Département des Sciences et Techniques de Productions Végétales, \\ BP 465, Maradi, Niger

\section{Dan Guimbo Iro} \\ Université Abdou Moumouni de Niamey, Faculté d'Agronomie, \\ Département de Génie Rural-Eaux Forêts \\ BP 10960, Niamey, Niger

\section{Toudou Daouada Abdoul Karim \\ Mahamane Ali} \\ Saadou Mahamane \\ Université Abdou Moumouni de Niamey, Faculté des Sciences et \\ Techniques, Département de Biologie Laboratoire Garba Mounkaila, \\ BP 10662, Niamey, Niger
}

\section{doi: 10.19044/esj.2017.v13n21p251 URL:http://dx.doi.org/10.19044/esj.2017.v13n21p251}

\begin{abstract}
This study is on the seeding and the initial growth of a food species, Diospyros mespiliformis, in Niger. Diospyros mespiliformis is an overexploited food species in Niger. The aim of this study is to determine the optimal conditions for germination and seedling growth of this species. In doing so, seed germination tests were conducted in petri dishes laboratory and seedling growth survey respectively. Also, the nursery was done using bags. The treatments involve the seed staying in warm water for 1, 3, 5, 7 days; scalding; and mechanical scarification. In the nursery, the substrates used are alluvial soil and alluvial soil mixed in $1 / 4,1 / 3$, and $1 / 2$ of organic
\end{abstract}


manure. As a result, the growth monitoring is done on plants kept in the shade. Others were exposed to the sun for 12 months by applying water stress (watering every day; 2 and 1 times per week; and without watering). Thus, the germination capacity is $55,4 \%$ without treatment and it reached $74 \%$ by scalding. The conservation makes it reduce to $43 \%$ after 6 months and $25 \%$ after 1 year. In the nursery, the emergence rate is $52,8 \%$ in alluvial soil, but it reduced to $10 \%$ in the substrate (soil $1 / 2$ and $1 / 2$ fertilizers). $D$. mespiliformis growth is slow during cold weather and becomes fast during hot weather. When the weather is shady or sunny, this species supports four months of drought by developing a significant root system. D. mespilformis can be used for afforestation.

Keywords: Diospyros mespiliformis, germination, growth, water stress, Niger

\section{Résumé}

Diospyros mespiliformis, fait partie des espèces les plus exploitées au Niger. L'objectif de cette étude est de connaitre, chez cette espèce, les conditions optimales pour la germination des graines et la croissance des plantules. Des tests de germination ont été réalisés aussi bien dans les boites de pétri au laboratoire que dans des pots en pépinière. En plus, les traitements pré-germinatifs effectués avec les séjours 1, 3, 5 et 7 jours de graines dans l'eau; l'ébouillantage et la scarification mécanique. En pépinière, les substrats utilisés sont le sol alluvial uniquement et le sol alluvial mélangé en proportion de volume à $1 / 4 ; 1 / 3$ et $1 / 2$ de fumure organique. Ensuite, le suivi de croissance est réalisé sur des plantes mises sous l'ombre et d'autres exposées au soleil pendant 12 mois en appliquant des stress hydriques (arrosées chaque jour; 2 fois et 1 fois par semaine; sans arrosées). Ainsi, sans traitement, le taux de germination est de 55,4\% et de $74 \%$ par ébouillantage. La conservation le fait ramener à 43\% après 6 mois et à $25 \%$ après $1 \mathrm{an}$. En pépinière, le taux de levée est de $52,8 \%$ en sol alluvial mais minoré à $10 \%$ dans le substrat ( $1 / 2$ sol alluvial et $1 / 2$ fumure). L'accroissement de $D$. mespiliformis est lent en période froide et devient rapide quand il fait chaud. A l'ombre ou exposée au soleil, cette espèce supporte quatre mois de sècheresse et elle développe un important système racinaire. D. mespilformis peut être utilisé dans les programmes de reboisement.

Mots-clés: Diospyros mespiliformis, germination, levée, stress hydrique, Niger 


\section{Introduction}

L'exploitation des plantes ligneuses fait partie des pratiques humaines les plus anciennes en milieu rural. Au Sahel, la récurrence des sècheresses combinée aux actions anthropiques influe négativement sur les essences forestières très prisées par la population rurale. L'exploitation incontrôlée des formations végétales boisées entraine la régression rapide, voire la disparition totale de certaines plantes très utiles aux communautés (Yossi et al., 2006). D. mespilformis fait partie des plantes ligneuses à usages multiples (Belemtougri et al., 2014). Au Niger, cet arbre fait partie des 32 espèces spontanées à grande utilisation (FAO, 2007) et ses fruits comestibles constituent un complément alimentaire en milieu rural (Adewale et al., 2014). Son utilisation en pharmacopée traditionnelle est bien connue par la population locale (Ngozi et al., 2015). Bien que rencontrée le long des certaines rivières et dans des dépressions ou des bas-fonds des zones sahélienne et soudanienne, $D$. mespilformis est l'une des espèces du genre Diospyros qui présente une large distribution géographique en Afrique subsaharienne (El-Kamali, 2011). Cependant, les travaux de (Saâdou, 1998) ont montré que cette espèce subie une forte pression principalement du fait de son utilisation. D'où la nécessité d'accompagner les initiatives des paysans dans son exploitation. Pour restreindre son érosion et la restaurer, sa domestication s'avère nécessaire. Ainsi, la maitrise de la germination et production des plantes en pépinière peut permettre de régénérer l'espèce. En effet, pour obtenir des populations de semis mieux adaptés à des conditions de croissance spécifiques, il est possible de les cultiver à partir de graines recueillies auprès d'arbres mères soigneusement sélectionnés (CTA, 1998).

L'objectif de ce travail est d'étudier la germination et la croissance en pépinière de $D$. mespiliformis. De manière spécifique il s'agit: d'évaluer les performances de la germination et de levée; suivre la croissance dans les différentes conditions et établir un seuil de tolérance à la sècheresse de l'espèce.

\section{Materiel et methodes \\ Sites}

Les tests de germination ont été menés au laboratoire de Biologie Garba Moukaila de la Faculté des Sciences et Technique de l'Université Abdou Moumouni de Niamey et le suivi de croissance des plantules au jardin botanique de ladite Faculté (altitude $188 \mathrm{~m}, 13^{\circ} 30^{\prime}$ Nord et $02^{\circ} 05^{\prime}$ Est).

\section{Provenance des Semences}

Les fruits ont été récoltés en janvier 2015 dans cinq localités différentes du Niger, constituants cinq lots de semences dont P1 pour 
Gazaoua, P2 pour Madarounfa, P3 pour Say ; P4 pour Torodi et P5 pour Téra dont la masse de 100 graines a été déterminé pour chaque provenance $(\mathrm{P} 1=33,2 \mathrm{~g}, \mathrm{P} 2=27 \mathrm{~g}, \mathrm{P} 3=40 \mathrm{~g}, \mathrm{P} 4=38,2 \mathrm{~g}$ et $\mathrm{P} 5=32,9 \mathrm{~g})$. Les fruits ont été récoltés à maturité et séchés à l'ombre pendant 3 semaines puis stockés dans des sacs en tissus. La figure 1 présente quelques aspects de ces différentes étapes.
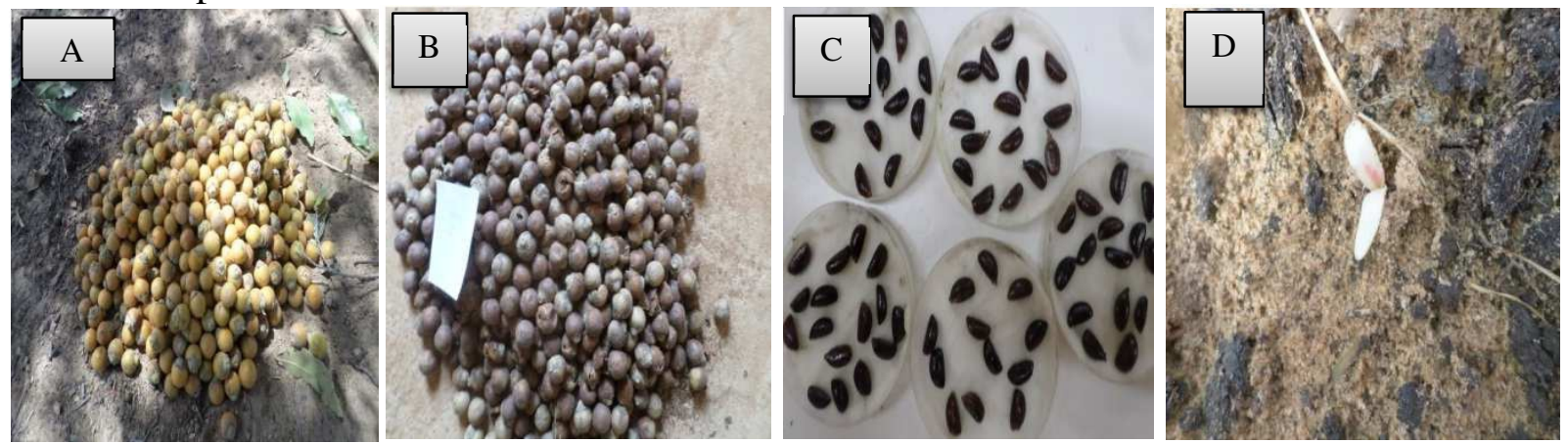

Figure 1. Baies mures (A), baies séchées (B), graines germées (C), graine levée(D)

\section{Germination}

Avant chaque test, les fruits sont minutieusement décortiqués à la main pour extraire les graines. Pour prendre en compte la différence entre les graines, le choix de celles-ci est fait au hasard et la germination est faite au laboratoire dans des boites de pétri de $5 \mathrm{~cm}$ de diamètre. Pour laisser un espace vide de moins 2 fois le volume des graines (ISTA, 2009), vingt graines ont été mise dans chaque boite de pétri. Cela permet de réduire la contamination d'une graine à l'autre. Le premier test de germination a eu lieu entre février et avril 2015 et a était appliqué sur 100 graines de chaque provenance soit un total de 500 graines (Figure 1B). Pour des espèces à graines dures, la germination peut être entravée par la dormance due notamment à l'imperméabilité des téguments ou du péricarpe à l'eau (Rollin, 1966). Pour améliorer le taux de germination nous avons utilisé les résultats $\mathrm{du}$ premier test. Ainsi la provenance qui présente un faible taux de germination a été utilisée pour l'application des traitements prégerminatifs. Les prétraitements testés sont décrits ci-après:

$>\mathrm{T} 0=$ Témoin : graines sans traitement préalable;

$>\quad \mathrm{T} 1=$ scarification mécanique $(\mathrm{Sc})$ des graines à l'aide d'un scalpel. Ce prétraitement a consisté à inciser le tégument de la graine sur une face dans le sens de la largeur;

$\mathrm{T} 2$ = ébouillantage $(\mathrm{Eb})$ : les graines ont été portées à ébullition pendant cinq minutes dans une quantité d'eau mesurant 2 fois le volume des graines puis l'ensemble (graines + eau chaude) est laissé à la température ambiante jusqu'au refroidissement; 
T3 : Trempage des graines dans l'eau tiède durant 1 jour;

T3 : Trempage des graines dans l'eau tiède durant 3 jours;

T5 : Trempage des graines dans l'eau tiède durant 5 jours;

T6 : Trempage des graines dans l'eau tiède durant 7 jours.

Les graines traitées et non traitées ont été mises à germination. Le test a été réalisé du 24 juin au 12 août 2015 dans des boîtes de pétri tapissées à leur fond d'un papier filtre à café, servant de rétenteur d'humidité (Photo 1). Afin d'éliminer les spores des champignons, avant chaque test, les graines sont trempées pendant 10 minutes dans une solution à $1 \%$ d'hypochlorite de sodium ( Rao, 2006) puis rincées à l'eau distillée et traitées avec le thirame (fongicide). Durant toute la durée de l'expérimentation, les papiers sont humectés régulièrement à l'eau distillée jusqu'à leur capacité de rétention toutes les 48 heures. Chaque prétraitement a été appliqué sur 100 graines qui sont reparties dans 5 boites de pétri à raison de 20 graines par boîte. Ces boîtes ont été disposées dans un germoir et à des températures qui varient entre $28^{\circ} \mathrm{C}$ et $31^{\circ} \mathrm{C}$ et qui correspondent aux normes préconisées pour les espèces tropicales et semi-tropicales. La sortie de la radicule est retenue comme critère de début de germination (CTA, 1998) et le suivi de germination était réalisé quotidiennement. Chaque graine germée est comptée puis retirée de la boîte.

\section{Longévité des Semences}

Pour tester la longévité d'une semence, la méthode la plus adéquate est le test de germination (Rao, 2006). Ainsi, pour tester la viabilité et la capacité de germination des semences, trois essais de germination sont réalisés à des intervalles réguliers de 6 mois. Le premier test réalisé juste après la récolte en janvier qui permettra de tester si l'espèce présente une dormance embryonnaire. Le deuxième test a eu lieu 6 mois après la récolte, correspondant à l'installation de la saison pluvieuse au Niger. En fin, le dernier essai était intervenu 12 mois après la récolte correspondant à une nouvelle saison sèche. Ce qui peut être un indicateur pour savoir si les graines peuvent résister pendant deux saisons de suite. Pour chaque test, 100 graines ont été prélevées du stock et mises en germination dans 5 boites de pétri.

Les paramètres calculés pour chaque test de germination sont :

$>\quad$ le taux de germination: (Nombre de graines germées/Nombre de graines mises à germer) x 100 ;

$>\quad$ la durée de germination (en jours): durée entre la première et la dernière germination;

$>\quad$ le délai de germination (en jours): durée entre le semis et la première germination. 


\section{Suivi de la levée}

Pour suivre la levée, une pépinière a été mise en place. Les semis d'un échantillon de 100 graines par provenance soit 500 graines ont été retenues pour l'étude des caractéristiques de la levée à la parcelle. En plus, pour étudier l'effet de substrat un autre échantillon de 400 graines était utilisé soit 100 graines par traitement. Les essais ont été réalisés dans des sacs servant de germoir contenant comme substrat du sol alluvial avec 20 graines par sac (Figure 1C). Les graines ont été semées à une profondeur égale à 2 fois leur diamètre (CTA, 1998). Une graine est considérée comme levée lorsque son coude hypocotylaire émerge hors du terreau (Ouédraogo et al., 1991). Le suivi de la levée a été fait quotidiennement et les paramètres calculés sont : le temps de latence; taux de levée et la durée de levée. Le dispositif d'étude de substrat était en quatre blocs présentés comme suit:

$\checkmark \quad$ bloc 1 : sol alluvial uniquement;

$\checkmark \quad$ bloc $2: 1 / 4$ de fumure et $3 / 4$ de sol alluvial;

$\checkmark \quad$ bloc $3: 1 / 3$ de fumure et $2 / 3$ de sol alluvial;

$\checkmark \quad$ bloc $4: 1 / 2$ de fumure et $1 / 2$ de sol alluvial.

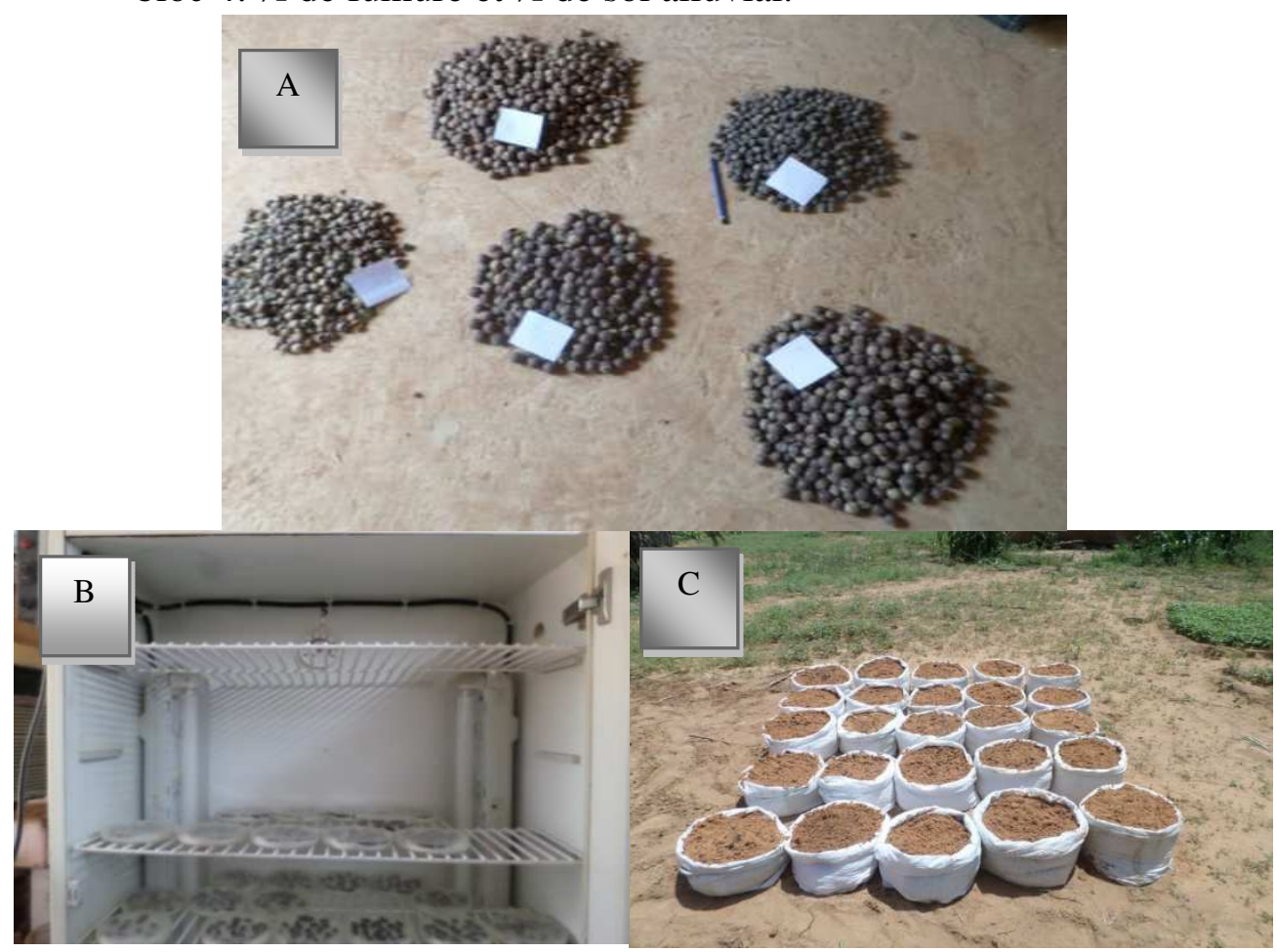

Figure 1. les fruits séchées (A), germination au laboratoire (B), germination en pépinière

(C) 


\section{Croissance des plantules}

Un échantillon de 210 plantules a été transplanté dans 210 rhizotrons pour le suivi de croissance. Ces rhizotrons utilisés sont des sacs (à l'ombre) et des pots (en plein soleil) pour tenir compte des effets des radiations solaires. Ces plantules sont âgées de 2 semaines et sont composées chacune de 2 à 3 feuilles. Les sacs et les pots ont les mêmes dimensions $(70 \mathrm{~cm}$ de hauteur et $30 \mathrm{~cm}$ de diamètre). L'arrosage est quotidien avec 5 litres par rhizotron. Ainsi, dans chaque rhizotron une plantule est repiquée pour éviter la compétition. Sur chaque plantule, le diamètre du collet, le nombre de feuilles par tige et la hauteur totale ont été mesurés chaque semaine.

Pour l'étude de stress hydrique, après la saison de pluies, deux (2) dispositifs composé de 4 blocs chacun ont été mis en place. L'un est directement exposé aux rayons du soleil et l'autre à l'ombre de Balanites aegytiaca (Figure 2). Chaque bloc comprend 10 plants âgés de 4 mois soit 80 plantules au total. Ces dispositifs permettent de vérifier si l'eau est un facteur limitant pour la croissance et de simuler le seuil de tolérance à la sècheresse de cette espèce dans son milieu écologique. Ainsi, le Bloc 1 est arrosé chaque jour, le Bloc 2 deux fois par semaine, le Bloc 3 une fois par semaine et le Bloc 4 est resté sans arrosage.

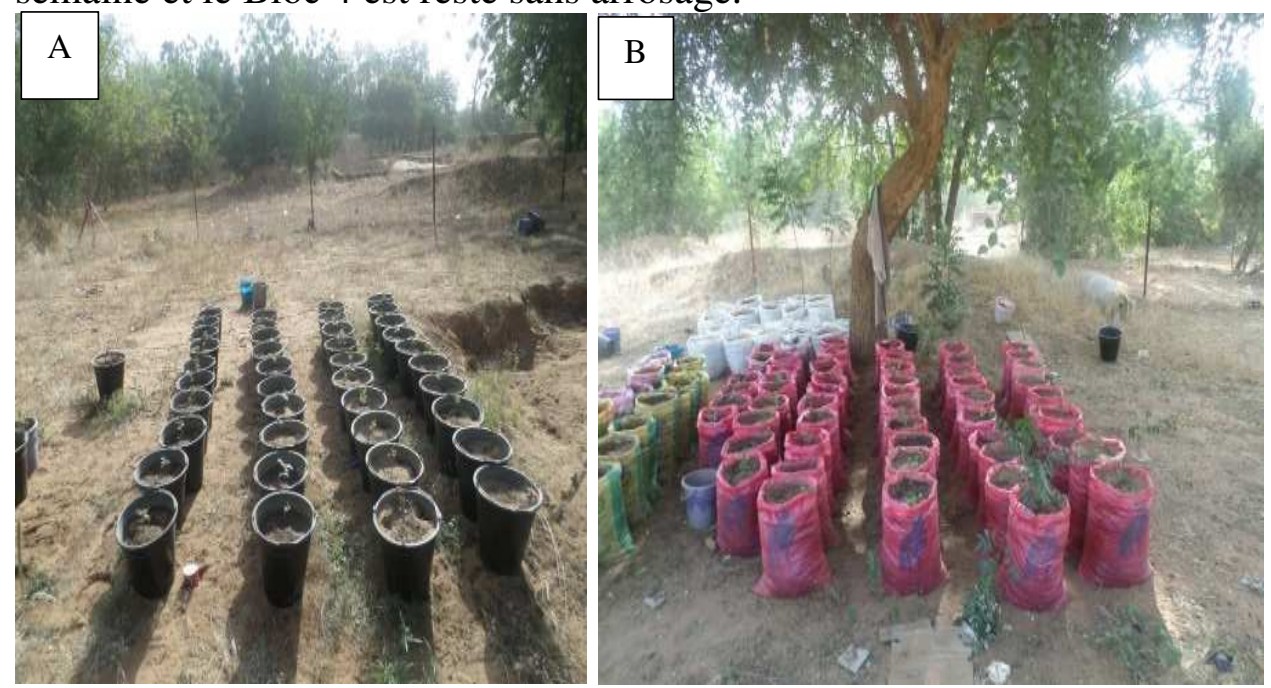

Figure 2. Dispositif de suivi de croissance des plantes (A) exposées au soleil et (B) à l'ombre.

Pour le suivi annuel de la croissance un échantillon de 100 plantules dont 50 à l'ombre et 50 exposées au soleil a été utilisé. La hauteur de chaque plantule a été mesurée une fois par semaine et a permis de calculer le taux d'accroissement relatif (TAR) selon la formule (Castel \& Terradas, 1994):

$$
\mathrm{RGR}=[\ln (h \mathrm{~T} 2)-\ln (h \mathrm{~T} 1)] /(\mathrm{T} 2-\mathrm{T} 1)
$$


A partir de cette formule, le taux d'accroissement relatif en nombre de feuilles a été déduit :

$$
\mathrm{RGR} n f=[\ln (n f \mathrm{~T} 2)-\ln (n f \mathrm{~T} 1)] /(\mathrm{T} 2-\mathrm{T} 1)
$$

avec $h$ la hauteur exprimée en centimètre, $n f$ le nombre de feuilles et $\mathrm{T}$ le temps en semaine

Un échantillon de 30 plantes a été utilisé pour le calcul de biomasses aériennes et souterraines car $D$. mespiliformis dispose un système racinaire échasses difficile a mesuré le pivot en fin de comparer avec la hauteur de tige comme l'avait décrit par (Laouali et al., 2014) sur Prosopis africana . Ainsi la masse fraiche (MF) et la masse sèche (MS) ont été pesées avec une balance électronique de marque SARTORIUS et de précision 1/1000. Ces mesures ont été effectuées sur des parties aériennes et des parties souterraines des plantules âgées de 3 mois, 6 mois et 12 mois après le semi. La masse fraiche a été mesurée directement après la récolte des plantules. Ensuite, les plantules ont été séchées à l'étuve à l'étuve à $70{ }^{\circ} \mathrm{C}$ pendant 3 jours pour mesurer la masse sèche. La teneur en eau (TE) a été calculée à l'aide de la formule suivante:

$$
\mathrm{TE}=(\mathrm{MF}-\mathrm{MS}) \times 100 / \mathrm{MF}
$$

\section{Analyses des Données}

Une analyse de variance (ANOVA, logiciel Minitab. 16) à un facteur a été effectuée pour évaluer les effets de la provenance, des prétraitements, l'environnement (ombre-soleil) et du régime hydrique sur les paramètres de germination et de croissance des plantules. Leurs moyennes ont été comparées à l'aide du test de Tukey et de Fisher au seuil de probabilité de $5 \%$.

\section{Resultats}

\section{Germination}

D. mespiliformis a présenté un taux moyen de germination de 55,4\% avec délai moyen de germination de 8 jours et temps moyen de germination de 20 jours et un faible coefficient de variation (Tableau 1). La comparaison des proportions entre les différentes provenances a montré que celle de Torodi avait un taux de germination significativement différent des autres provenances.

Tableau 1. Le délai, le taux et le temps de germination chez D. mespiliformis pour chaque

\begin{tabular}{|c|c|c|c|}
\hline Provenance & Taux de germination (\%) & Délai de germination & Temps de germination \\
\hline $\mathrm{p} 1$ & $54 \mathrm{a}$ & 7 & 23 \\
\hline p2 & $45 \mathrm{a}$ & 9 & 20 \\
\hline p3 & $60 \mathrm{a}$ & 9 & 18 \\
\hline p4 & $75 b$ & 7 & 19 \\
\hline
\end{tabular}
provenance 
$43 \mathrm{ac}$

8

20

Moyenne

$55,4 \pm 12,93$

$8 \pm 1$

$20 \pm 1,87$

CV (\%)

23,33

12,5

9,35

Les moyennes ne partageant aucune lettre sont sensiblement différentes

Le Tableau 2 présente le délai, le taux et le temps de germination en fonction des prétraitements germinatifs. L'ébouillantage a augmenté le taux de germination ( $74 \%$ contre $43 \%$ pour le témoin) et a réduit le temps de germination de 2 jours. Les séjours des graines dans l'eau tiède pendant 1 jour et 2 jours n'ont pas eu d'effet sur le taux de germination et le délai de germination mais ont réduit le temps de germination de 6 et de 7 jours respectivement. Le séjour pendant 7 jours a réduit significativement le taux et le temps de germination mais a augmenté le délai de germination de 4 jours. Tous les prétraitements germinatifs ont réduit le temps de germination. Le test de comparaison de Fisher a montré que l'ébouillantage, les séjours dans l'eau (5 et 7 jours) étaient significativement différents du témoin et les autres traitements.

Tableau 2. Le délai, le taux et le temps de germination en fonction des prétraitements germinatifs

Traitements Taux de germination (\%) Délai de germination Temps de germination

\begin{tabular}{llcc}
\hline T0 & $43 \mathrm{~b}$ & 8 & 20 \\
T1 & $35 \mathrm{~b}$ & 9 & 16 \\
T2 & $74 \mathrm{a}$ & 9 & 18 \\
T3 & $44 \mathrm{~b}$ & 7 & 14 \\
T4 & $44 \mathrm{~b}$ & 7 & 13 \\
T5 & $18 \mathrm{c}$ & 13 & 9 \\
T6 & $2 \mathrm{~d}$ & 12 & 1 \\
\hline
\end{tabular}

Les moyennes ne partageant aucune lettre sont sensiblement différentes

\section{Levées de Germination en Pépinière}

Chez D. mespiliformis, le taux de levée moyen était de 52,8\% avec un délai et une durée de germination respectivement de 9 et 27 jours (Tableau 3). Le test de comparaison de proportion de Fisher a montré des différences selon les provenances. Le taux de levée était significativement plus élevé pour la provenance de Torodi (P4) mais significativement plus faible pour la provenance de Téra (P5).

Tableau 3. Le délai, le taux et temps de levés selon les provenances

\begin{tabular}{cccc}
\hline Provenance & Taux de levée $(\%)$ & Délai de levée (jours) & Temps de levée (jours) \\
\hline P1 & $54 \mathrm{~b}$ & 7 & 29 \\
P2 & $40 \mathrm{~b}$ & 10 & 26
\end{tabular}




\begin{tabular}{cccc} 
P3 & $57 \mathrm{~b}$ & 10 & 26 \\
P4 & $77 \mathrm{a}$ & 10 & 26 \\
P5 & $36 \mathrm{c}$ & 10 & 26 \\
\hline Moyenne & $52,8 \pm 16,2$ & $9,4 \pm 1,34$ & $26,6 \pm 1,34$ \\
CV $(\%)$ & 30.70 & 14.27 & 5.04 \\
\hline
\end{tabular}

Les moyennes ne partageant aucune lettre sont sensiblement différentes

Le taux de levée a diminué significativement avec la teneur élevée de fumure organique. Le taux de levée le plus élevé a été observé au niveau de substrat contenant uniquement le sol alluvial (77\%) et le plus faible dans $1 / 2$ de sable et $1 / 2$ de fumure (10\%) (Tableau 4$)$.

Tableau 4. Le délai, le taux et temps de levés de germination chez D. mespiliformis selon les traitements.

\begin{tabular}{cccc}
\hline Traitement & Taux de levée (\%) & Délai de levée (jours) & Temps de Levée (jours) \\
\hline S0 & $77 \mathrm{a}$ & 31 & 10 \\
S1 & $27 \mathrm{~b}$ & 29 & 10 \\
S2 & $17 \mathrm{c}$ & 28 & 8 \\
S3 & $10 \mathrm{c}$ & 26 & 5 \\
\hline
\end{tabular}

S0 sol alluvial; $\mathrm{S} 1$ : $1 / 4$ de fumure et $3 / 4$ de du sable alluvial ; S2 : $1 / 3$ de fumure et $2 / 3$ sol alluvial; S3 $1 / 2$ de fumure et $1 / 2$ de sol alluvial Les moyennes ne partageant aucune lettre sont sensiblement différentes

\section{Longévité du Pouvoir Germinatif}

Le taux et le délai de germination ont diminué avec l'allongement de la durée de conservation tandis que le temps de germination a augmenté

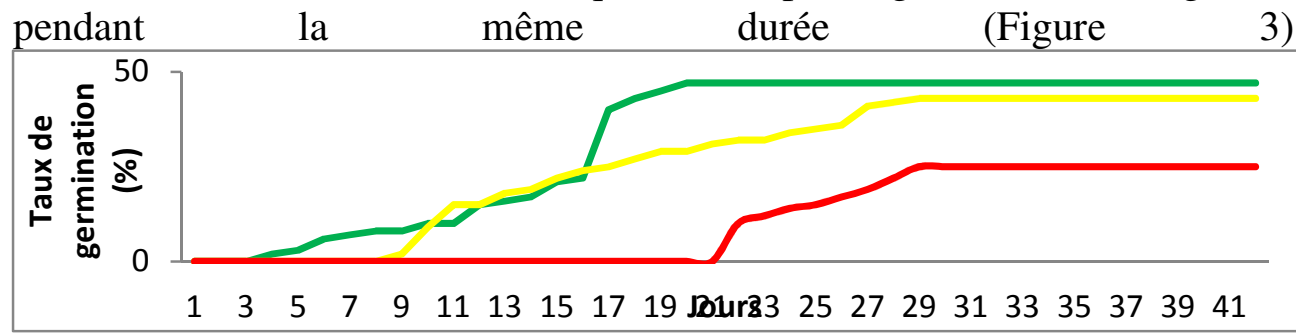

Figure 3. Courbes d'évolution de délai, du taux et du temps de germination des graines de D. mespiliformis suivant la durée de conservation (tiret vert germination juste après la récolte, tiret jaune 6 mois après la récolte et tiret rouge un an après la récolte).

\section{Croissance}

De la levée jusqu'au 114ème jour, la hauteur était de 11,84 cm lorsque les plantules sont exposées au soleil et était $11,24 \mathrm{~cm}$ à l'ombre (Figure 4A). Le nombre de feuilles était de 8,42 à l'ombre est de 9,72 à 
l'ombre (Figure 4B). Le diamètre moyen avait atteint 2,37 $\mathrm{mm}$ à l'ombre et 2,45 $\mathrm{mm}$ pour les plantules exposées au soleil (Figure 4C).
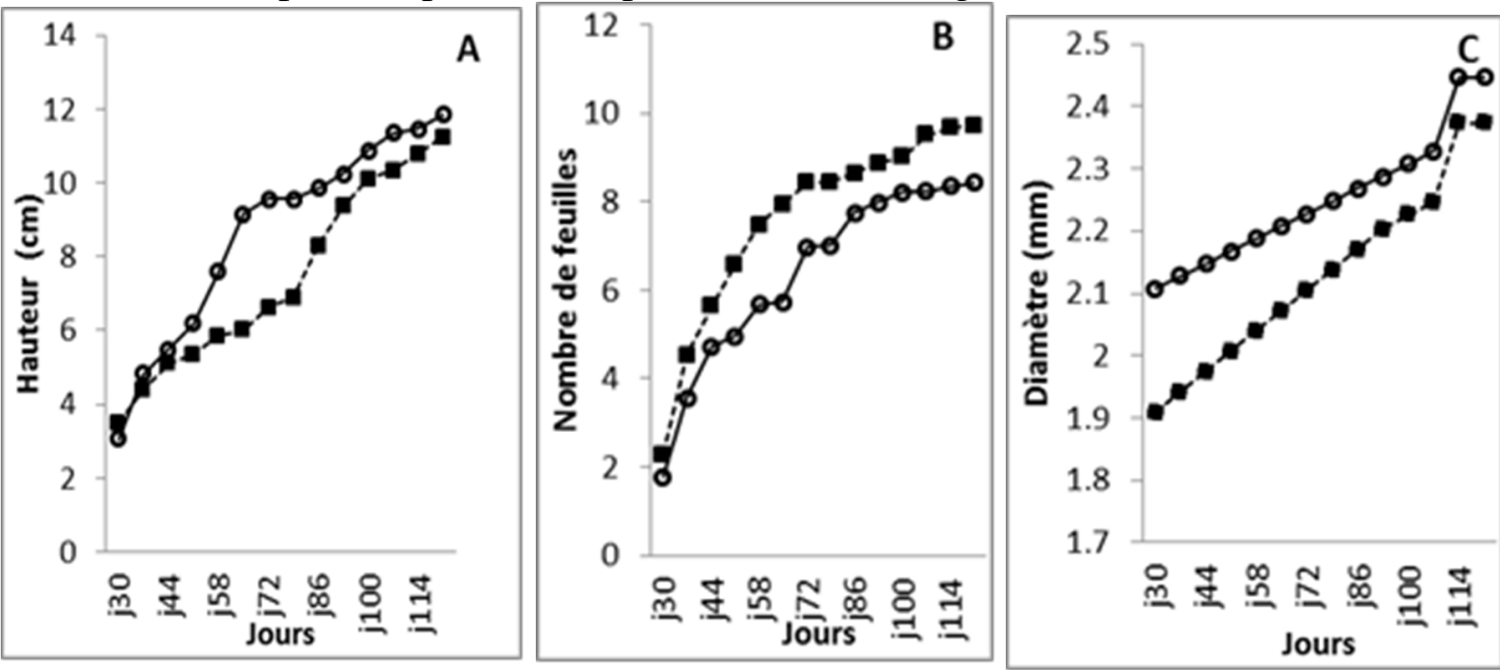

Figure 4. Evolution de la hauteur (A), du nombre de feuilles (B) et du diamètre (C) des plantules de D. mespiliformis pendant la saison pluvieuse. Les valeurs mesurées sur les plantules de l'ombre sont représentées par les carrés pleins et les plantules laissées au soleil par les cercles ouverts.

Après la saison pluvieuse, les plantes mises à l'ombre ou exposées aux rayons solaires avait un accroissement relatif lent. Dans ces conditions l'espèce a supporté 12 semaines de sécheresse. Pendant tout ce temps le taux d'accroissement relatif des plantes exposées aux rayons solaires est de : 0,02 $\mathrm{mm}$ pour T0; $0,013 \mathrm{~mm}$ pour $\mathrm{T} 1 ; 0,013 \mathrm{~mm}$ pour $\mathrm{T} 2$ et $0,005 \mathrm{~mm}$ pour T3. Pour les plantes mises à l'ombre l'accroissement relatif est de : $0,1 \mathrm{~mm}$ en T0; 0,004 $\mathrm{mm}$ en T1; 0,003 mm en T2 et $0,001 \mathrm{~mm}$ en T3. Celui-ci change avec une tendance réductionnelle en fonction de durée de sécheresse (Figure $5 \mathrm{~A} 1$ et 5B1). En outre, pendant toute la durée de l'expérience, le taux d'accroissement relatif en nombre de feuilles pour les plantes exposées au soleil était de 0,004 pour $\mathrm{T} 0 ; 0,018$ pour $\mathrm{T} 1 ; 0,004$ pour T2 et 0,005 pour T3 alors qu'il est de 0,013 pour T0 ; 0,011 pour T1 : 0,007 pour T2 et 0 pour T3. La tendance générale était régressive pour les plantules exposées au soleil (Figure 5A2) tandis qu'elle était évolutive pour les plantules mises à l'ombre (Figure 5B2). 


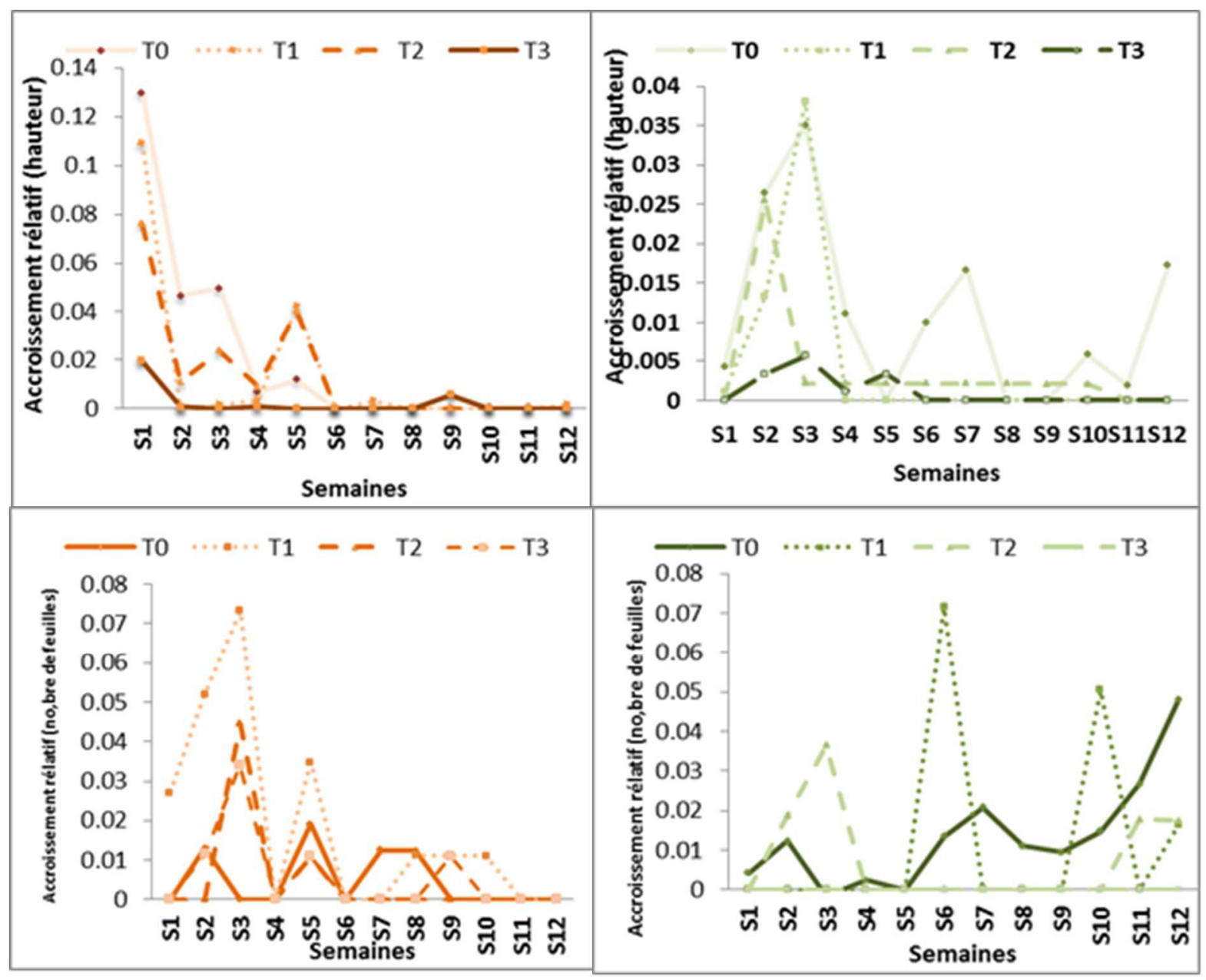

Figure 5. Variation du taux d'accroissement relatif (TAR) en fonction de la durée du stress hydrique et de la position des plantules (ombre, soleil) : TAR en hauteur pour les plantes exposées au soleil A1 et à l'ombre A2, TAR en nombre de feuilles pour les plantes exposées au soleil B1 et à l'ombre B2.

Pour plantules arrosées quotidiennement, le suivi pendant 12 mois montre que la plus importante croissance cumulée se faisait en deux phases chez D. mespilformis. La première phase intervenait pendant les mois d'août, septembre et octobre et la deuxième en mars, avril et mai (Figure 6). 

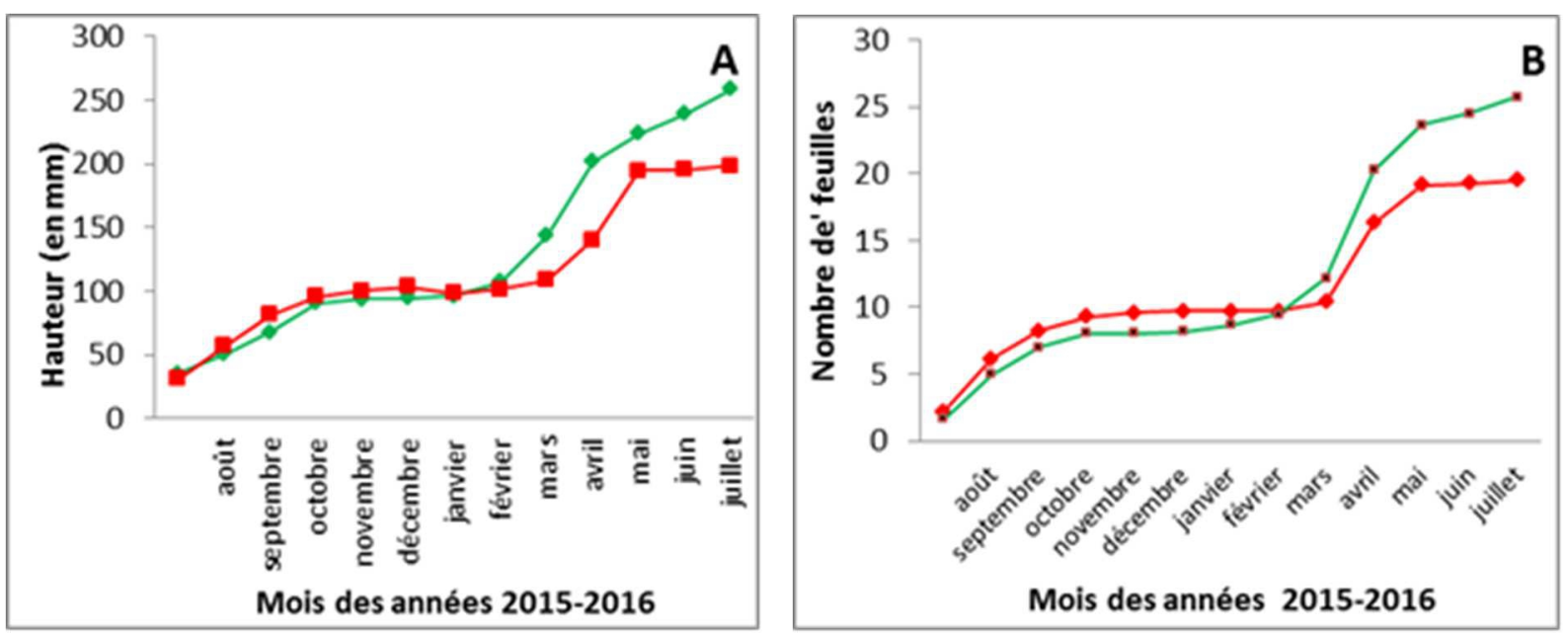

Figure 6. Courbes d'évolution de la croissance moyenne cumulée des plantes en fonction des mois (tiret vert à l'ombre, tiret rouge au soleil): (A) en hauteur et (B) en nombre de feuilles.

Le taux d'accroissement relatif était plus élevé en Août et plus bas en Décembre (Figure 7).
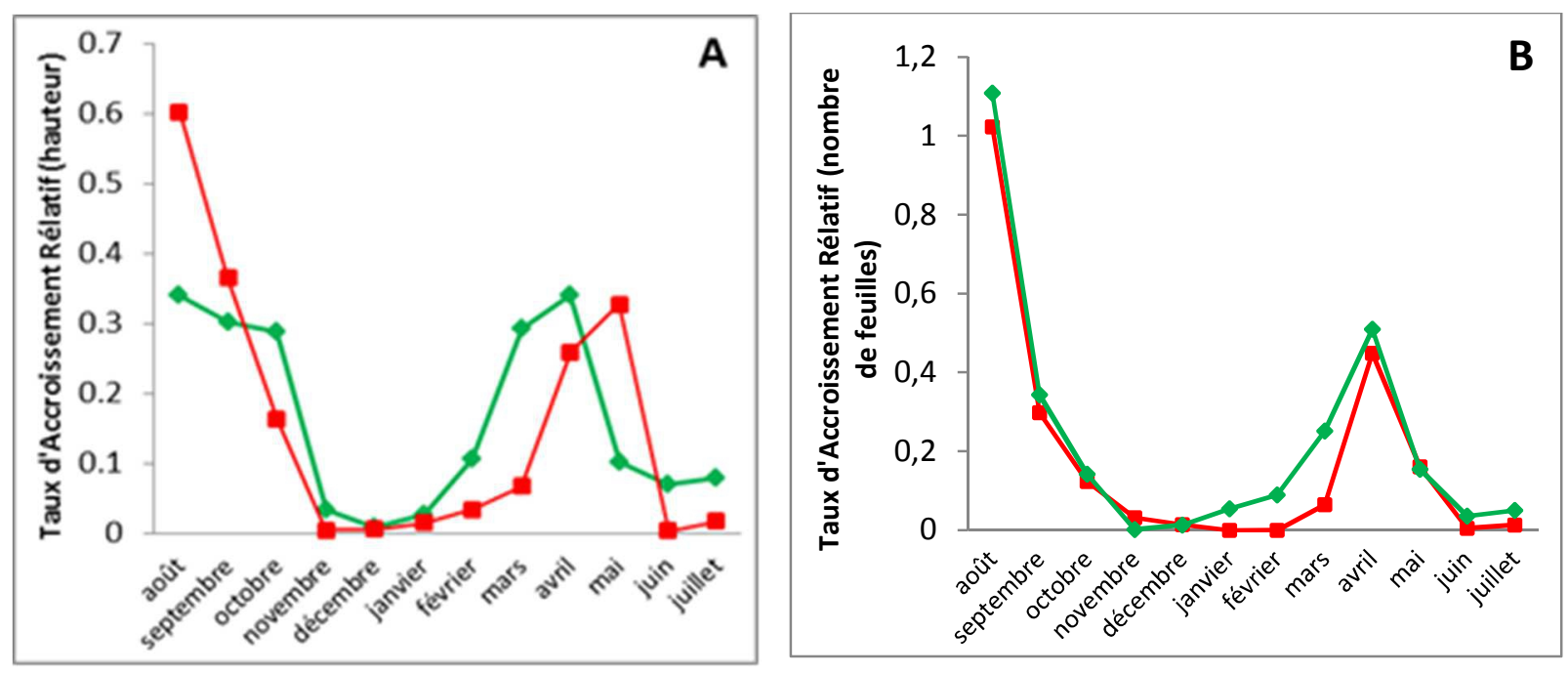

Figure 7. Variation du taux d'accroissement relatif en hauteur TAR (A) et en nombre de feuille TARnf $(\mathrm{B})$ chez D. mespiliformis en fonction de la situation (tiret vert à l'ombre, tiret rouge au soleil)

Une différence significative s'observe entre la partie aérienne et la partie souterraine qu'après 1 an de croissance pour les plantes sous l'ombre. La teneur en eau diminue de façon significative en fonction du temps, sauf 
pour la partie souterraine des plantes exposées aux rayons solaires (Tableau 5).

Tableau 5. Évolution de masse sèche et de la teneur en eau en fonction du temps.

\begin{tabular}{|c|c|c|c|c|c|c|}
\hline \multirow[b]{2}{*}{ Paramètre } & & 3 mois & \multicolumn{3}{|c|}{6 mois } & 12 mois \\
\hline & & sous l'ombre & au soleil & sous l'ombre & au soleil & sous l'ombre \\
\hline \multirow{5}{*}{ Masse sèche } & $\mathrm{P}$ & & & & & \\
\hline & A & $0,28 \pm 0,20 \mathrm{c}$ & $0,2 \pm 0,12 \mathrm{c}$ & $1,55 \pm 1,33 \mathrm{c}$ & $0,48 \pm 0,24 c$ & $10,82 \pm 2,11 b$ \\
\hline & PS & $0,47 \pm 0,25 \mathrm{c}$ & $0,24 \pm 0,15 \mathrm{c}$ & $3,12 \pm 1,75 \mathrm{c}$ & $0,42 \pm 0,16 \mathrm{c}$ & $15,56 \pm 5,07 \mathrm{a}$ \\
\hline & $\mathrm{P}$ & $74,83 \pm 12,39$ & & $71,83 \pm 12,48 a$ & $73,69 \pm 19,4 a$ & \\
\hline & A & $\mathrm{a}$ & $78,67 \pm 9,01 \mathrm{a}$ & $\mathrm{b}$ & $\mathrm{b}$ & $50,92 \pm 17,84 a$ \\
\hline $\begin{array}{c}\text { Teneur en } \\
\text { eau }\end{array}$ & PS & $\begin{array}{c}70,86 \pm 3,73 a \\
b\end{array}$ & $\begin{array}{c}70,29 \pm 5,81 \mathrm{a} \\
\mathrm{b}\end{array}$ & $76,11 \pm 1,92 \mathrm{a}$ & $76,38 \pm 4,59 a$ & $\begin{array}{c}61,03 \pm 12,26 a \\
b\end{array}$ \\
\hline
\end{tabular}

$\mathrm{PA}=$ partie aérienne et $\mathrm{PS}=$ partie souterraine. Les moyennes ne partageant aucune lettre sont sensiblement différentes

La Figure 6 montre la relation entre la hauteur et le nombre de feuilles et aussi entre la biomasse aérienne et la biomasse souterraine. La variabilité expliquée est plus élevée chez les plantules exposées aux rayons solaires $\left(\mathrm{R}^{2}=0.98\right)$ que celles à l'ombre $\left(\mathrm{R}^{2}=0.91\right)$. L'analyse de la courbe de variation montre surtout pour les plantes.
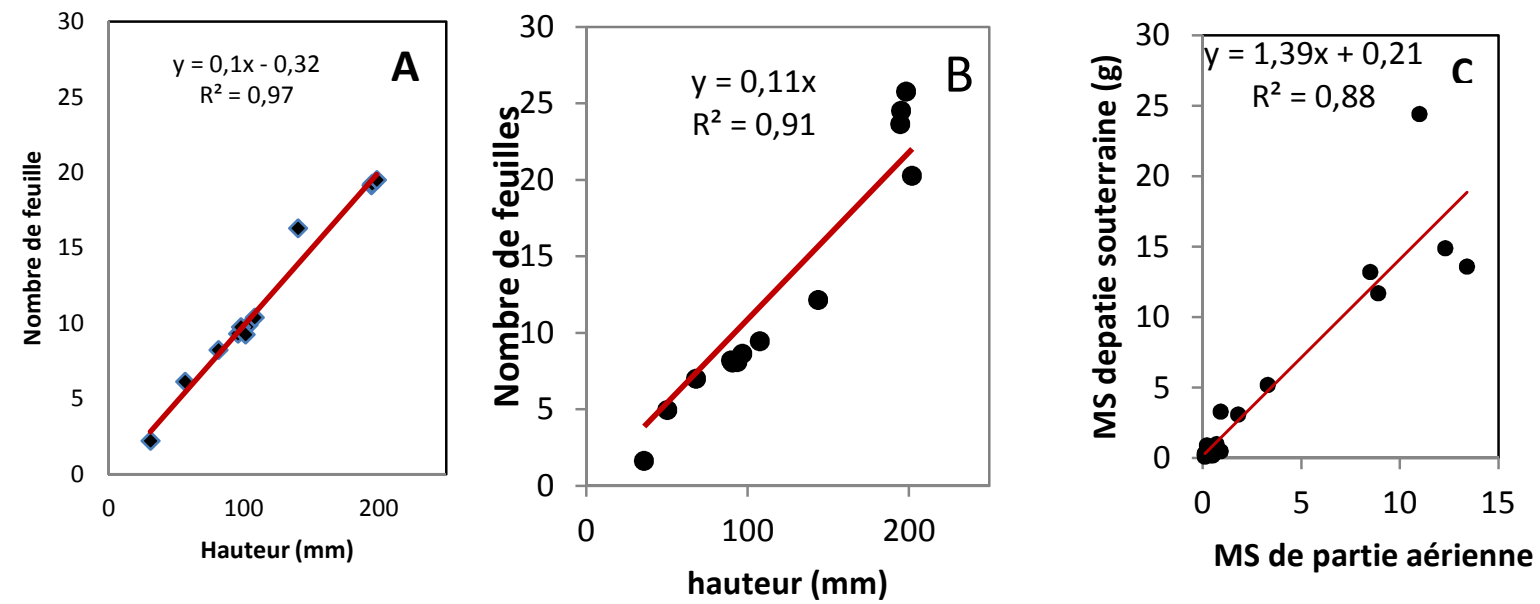

Figure 8. Régression entre la hauteur et le nombre de feuilles chez D. mispiliformis (A des plantules exposées au soleil, B plantules mises sous l'ombre) et régression entre biomasses sèches souterraine et aérienne $(\mathrm{C})$

\section{Discussion}

Les travaux réalisés au laboratoire montrent que le taux moyen de germination de D. mespiliformis est de 55,4\% donc relativement faible par rapport à celui de certaines espèces qui fréquentent le même milieu notamment Sclerocarya birrea (A. Rich.) Hochst qui présente un taux de germination de 68,33\% (Haminou et al., 2013) et les quatre combrétacées 
dont le taux de croissance varie de 70\%à100\% (Amani el al., 2015) donc nettement supérieur à celui de d'une espèce de même genre Diospyros lotus qui n'est que de 23,8\% (El-Refaey et al., 2014). Ce même taux est nettement amélioré à l'aide des techniques simples dont la plus prometteuse est l'ébouillantage qui l'améliore de l'ordre de 30\%. Le choc thermique est un moyen efficace pour ramollir le tégument des graines de la plupart des espèces (Rabaud L. \& Oustric J., 1989).Cette technique est très défavorable pour certaines espèces comme Neocarya macrophylla (Dan Guimbo et al., 2010) et Prosopis africana (Laouali et al.,2015). Ce résultat implique l'existence d'une dormance tégumentaire au niveau des graines de $D$. mespiliformis. La scarification mécanique, difficile à pratiquer à grande échelle, améliore peu le taux de germination. En outre le séjour dans l'eau contribue au pourrissement des graines. En pépinière, le délai de levée est assez long (19 jours). Cela est dû au temps mis par les radicules à traverser la couche de terre. Cette levée peut être un facteur limitant en milieu naturelle car les graines de fois trop enfoncées peuvent avoir des difficultés à surmonter la charge. Ce qui peut entrainer leur dessiccation ou leur causées des dommages irréversibles si les gemmules arrivent à lever. Les substrats est un facteur déterminant dans la levée de germination en pépinière. Le meilleur substrat pour la germination de D. mespiliformis est le sol alluvial. Tout mélange tend à réduire ce taux. Cela peut être un indicateur clé pour montrer que la température est un des paramètres déterminant pour la germination avec un optimum dans le substrat de sol de rivière donc à basse température.

La conservation à l'air libre réduit la faculté germinative même si l'obtention de $25 \%$ de graines germés donne espoir à ce que les stocks de semenciers puissent servir aux prochaines années. Les travaux de (Babou et al., 2000; Dan Guimbo et al., 2010; et Laouali et al., 2015) ont montrés que la condition de conservation ne modifie pas la viabilité pendant au moins un an en l'expliquant par la protection tégumentaire des graines de A. africa, $N$. macropylla et $P$. africana. Cependant pour $D$. mespiliformis les téguments ne sont pas très dures car ses graines ont un délai de germination de 3 jours pour les graines non conservées et atteignent 21 jours pour celle semées après un an. Des résultats similaires ont été trouvés par (Sidi et al., 2013) sur Carapa procera DC.

L'accroissement relatif est rhytmique chez D. mespiliformis. Il est globalement beaucoup plus important en Aout, Septembre, Octobre, Mars, Avril et Mai. Il atteint son pic en aout et avril. Ces différences peuvent être dues à l'augmentation des températures en Aout et Avril qui sont les mois les plus chauds au Niger. Ces phases d'accroissement sont observées par (Amani et al., 2015). L'une des caractéristiques du monde organique réside dans l'aspect rhytmique de nombreux processus de croissance et des 
structures qui en résultent même si certaines espèces semblent y répondre de façon opposée à d'autres (Ernst Zürcher, 1992). L'adaptation des espèces aux conditions sahéliennes, arides ou semi-arides consiste à un important développement du système racinaire pendant la première phase de croissance pour supporter la sècheresse. La phase d'accroissement nulle correspond aux activités internes des bourgeons et qui ne s'expriment presque pas par l'apparition des feuilles. La soumission aux stress hydriques même si elle ne montre pas une différence d'accroissement peut-être indicatrice de cette adaptation. D. mespiliformis supporte plus de trois de sécheresse lorsqu'elle

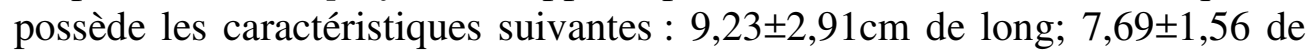
feuilles et 2,15+1,5 de diamètre à l'ombre et en plein soleil. Pendant cette période la croissance des parties aériennes est quasi-stationnaire.

Les biomasses aériennes et souterraines croissent pratiquement dans un même sens suivant les différentes périodes de l'année. Ce qui pourrait être un bon indicateur pour l'adaptation de l'espèce en conditions hygrométriques difficiles.

\section{Conclusion}

D. mespiliformis présente un faible taux de germination et il existe des techniques simples permettant d'améliorer ce taux, notamment l'ébouillantage. La longévité est réduite considérablement avec une longue durée de conservation. Le sol alluvial sans fumier organique constitue le meilleur substrat pour la germination en pépinière. Malgré une croissance lente au début, une longue durée en pépinière des plantules permet d'obtenir des plants suffisamment vigoureux pour être transplantés aux champs. Cette croissance se fait en plusieurs phases avec une phase de croissance exponentielle en période chaude. La durée d'arrosage a peu d'influence sur la croissance de l'espèce. Elle présente une grande capacité de tolérance à la sécheresse ce qui lui confère une adaptabilité aux irrégularités des pluies en milieu sahélien. Elle peut donc être utilisée dans le programme des reboisements en produisant dans des pépinières, des jeunes plants, juste après la récolte de graines mûres. La maitrise de la germination et production des plantes en pépinière constitue une démarche écologiquement et techniquement rentable pour la restauration des plantes ligneuses.

\section{References:}

1. Adewale A. \& Rotimi A. O. (2014). Fatty Acid Composition and Lipid Profile of Diospyros mespiliformis, Albizia lebbeck, and Caesalpinia pulcherrima Seed Oils from Nigeria. International Journal of Food Science; ID 283614, 6 pages 
2. Amani, M.M. Inoussa, I. Dan Guimbo, A. Mahamane, M. Saadou \& A.M. Lykke (2015) Germination et croissance de quatre espèces de Combretaceae en pépinière. Tropicultura, 33,2,135-145.

3. Arbonnier M. (2000). Arbres, arbustes et lianes des zones sèches d'Afrique de l'Ouest. CIRAD - MNHN - UICN, Montpellier (France), $541 \mathrm{p}$.

4. Adzu, S. Amos, S. Dzarma, I. Muazzam, \& K.S. Gamaniel (2002). Pharmacological evidence favouring the folkloric use of Diospyros mespiliformis Hochst in the relief of pain and fever. Journal of Ethnopharmacology ; 82 : 191_195.

5. Babou A. B, Sibiri J.O. \& Sita G (2000). Longévité des graines et contraintes à la survie des plantules d'Afzelia africana .Sm dans une savane boisée du Burkina Faso.Ann. For. Sci. 58 : 69-75

6. Belemtougri RG, Constantin B, Cognard C, Raymond G \& Sawadogo L. (2006). Effects of two medicinal plants, Psidium guajava L. (Myrtaceae) and Diospyros mespiliformis L. (Ebenaceae) leaf extracts on rat skeletal muscle cells in primary culture. J Zhejiang University Sci. 7(1): 56-63.

7. Charles O.E., Chukwuemeka S N., Ebere B O., Justina U O., Kelechi L N., Joy C O., Maria I N., \& Kennedy F.C. (2009). Antimycobacterial activity of root and leaf extracts of Anthocleista djalonensis (Loganiaceae) and Diospyros mespiliformis (Ebenaceae).

8. CTA (1998). Multiplier et planter des arbres sérieAgrodok 19. Wageningen, PaysBas.83.

9. Dan Guimbo I., Ambouta K.J.M., \& Mahamane A., M. (2011). Germination et croissance initiale de Neocarya macrophylla (Sabine) Prance, une espèce oléagineuse du Niger. Tropicultura, 29 (2): 88-93.

10. El-Refaey F. A. El-Dengawy \& Ahmed A. Hussein (2014).The effects of treating persimmon (Diospyros lotus) seeds with moistchilling and growth regulators on seeds germination, the subsequent seedling characters and their induced drought tolerance. IOSR. J of Agri and Vet Science 7 (9), PP 45-53

11. Ernst Zürcher (1992). Rythmicité dans la germination et la croissance initiale d'une espèce forestière tropicale. Schwiez Z. Forestwes, 143, 12 :951-966.

12. Hamidou A.,Dan Guimbo i, Morou B, Taffa S. M, \& Mahamane .A. (2013). Potential germination and initial growth of Sclerocarya birrea (A. Rich.) Hochst in Niger. Journal of Applied Biosciences 76:6433-6443.

13. ISTA (2009). Règles internationales pour les essais de semences. Bassersdorf, Suisse : Association internationale d'essais de semences (AIES). 
14. Laouali A, Dan Guimbo I, Youchaou A, Rabiou H. \& Mahamane A. (2015b). Etude de la germination de la graine et suivi de la croissance en pépinière de Prosopis africana (G. et Perr.) Taub., espèce menacée de disparition au Niger. Ann. Univ. Abdou Moumouni (Niger), Sciences ; (2015) ; 18-A pp 1-12.

15. Ngozi Justina Nwodo (2015). Anti-Trypanosomal Activity of Nigerian Plants and Their Constituents; Molecules 20, 7750-7771.

16. Rao NK, Hanson J, Dulloo ME, Ghosh K, Nowell D. \& Larinde M. (2006). Manuel de manipulation des semences dans les banques de gènes. Manuels pour les banques de gènes No. 8. Bioversity International, Rome, Italie.

17. Saadou M. (1990). La végétation des milieux drainés nigériens à l'Est du fleuve Niger: Thèse de Doctorat ès - Sciences Naturelles. Université de Niamey. 395 p.

18. Trabaud L. \& Oustric J. (1989). Influence du feu sur la germination des semences de quatre espèces ligneuses méditerranéennes à reproduction sexuée obligatoire. Seed science and technology, 17, 3, 589-599.

19. Yossi H. et al. (2006). Les haies vives au Sahel. État des connaissances et recommandations pour la recherche et le développement. Occasional Paper no. 6. Nairobi : World Agroforestry Centre (ICRAF). 